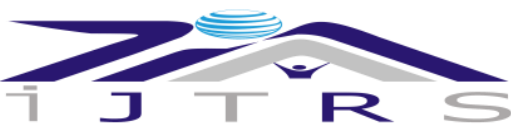

International Journal of Technical Research \& Science

\title{
INVESTIGATION AND CALCULATIONS OF RADIATION INTERACTION PARAMETERS OF SOME SHAPE MEMORY ALLOYS
}

\author{
Saniye Tekerek \\ E-Mail Id: saniyetekerek@ksu.edu.tr
}

Vocational School of Health Services, Kahramanmaraş Sutçu Imam University, Turkey

Abstract- In this study, the mass absorption coefficient $(\mu / \rho)$, half-value thickness (HVL), one-tenth thickness (TVL), mean free path ( $\lambda$ ) values were calculated of some shape memory alloys at 5.9-6.1-8-11.2-25-59.543-75-112-149 $\mathrm{keV}$ energy. It has been observed that the obtained values of the calculated parameters vary depending on the photon intensity, chemical constitution and density of the alloys. Calculations were made using the WinXCOM program and the graph of the change according to the energy of the obtained results was drawn. The results of this study are thought to be beneficial in the application of various fields.

Keywords: Half-value thickness, Mass absorption coefficient, Mean free path.

\section{INTRODUCTION}

In the last decade, smart materials and structures have gained increasing attention due to their enormous technological and scientific results. Shape memory alloys (SMA) are functional materials that exhibit unique thermo-mechanical properties such as shape memory effect and flexibility. SMA alloys are mostly used in medical fields such as stents and surgical instrument manufacturing etc. [1].

As absorption, penetration and photon interactions with the substance are important in every field, their effects and knowledge on SMA are very important. Photon interactions have been widely used in many fields such as medicine, industry, agriculture, nuclear technology, and space research [2]. Studies on the absorption properties of gamma rays in composite materials have become an interesting research area [3]. Linear, mass attenuation coefficients, effective atomic number, effective electron number, half value layer, tenth value layer and mean free path are the basic parameters for the penetration and absorption properties of $\mathrm{x}$-ray or gamma rays. These parameters have an important place in many different fields such as radiation protection, nuclear diagnosis and therapy, nuclear medicine and radiation dosimetry. The exact values of the mass absorption coefficient occupy a great place in researches to solve diverse problems in radiation physics and chemistry [4].

Shape memory alloys that can change their shape under the applied temperature or conditions since materials attract great attention from the technological point of view. Thanks to its superior functional properties such as shape memory effect, super elasticity, high absorption ability, corrosion resistance and biocompatibility seen in such materials, it has a widespread use potential in many industrial and medical applications [5]. Although it is known that there are many alloys that show shape memory effect in various application areas, especially in the field of medicine, one of the most popular among these alloys is iron-based shape memory alloys [6]. Shape memory alloys are materials that are being researched and developed for use in a variety of applications in multidisciplinary fields such as space research, medicine, and micro electromechanics. One of the most important factors in choosing SMA materials is that the compatibility of the material with the environment gives good results. It is important to investigate the nature of this material in order to make suitable designs for the purpose of use in applications [7].

Various studies are carried out in different energy ranges in order to determine various radiation interaction parameters on element, compound, mixture and alloy. In many studies in the literature, the linear, mass attenuation coefficient and other absorption parameters of alloys, amino acids and dosimetric materials were carried out with high energy photons and examined the reaction of the materials to radiation. [8-12]. 
7 J 1 R 5 International Journal of Technical Research \& Science

It has been observed that there is not much room for theoretical data on the study of the absorption properties of shape memory alloys in the literature. The main purpose of this study is to calculate mass absorption coefficients and other relevant parameters at 5.6-6.1-8-11.2-25-59.543-75-112-149 keV energy using WinXCOM program. In the light of the obtained data, it is thought that this study will contribute to the literature, since it will be possible to understand the behavior of some SMAs against high-energy photons.

\section{MATERIAL AND METHOD}

The mass attenuation coefficient $(\mu / \rho)$ is independent of chemical, physical properties and dependent on energy [13]. The mass absorption coefficient was calculated using Equation (1).

$$
\left(\frac{\mu}{\rho}\right)_{\text {Alloy }}=\sum_{\mathrm{i}} \omega \mathrm{i}\left(\frac{\mu}{\rho}\right)_{\mathrm{i}} ; \quad \omega_{\mathrm{i}}=\frac{\mathrm{a}_{\mathrm{i}} \mathrm{A}_{\mathrm{i}}}{\sum \mathrm{a}_{\mathrm{i}} \mathrm{A}_{\mathrm{i}}}
$$

In Equation (1), $\rho$ is the density of the alloys, $\omega_{\mathrm{i}}$ is the atomic fraction of the element, $(\mu / \rho)_{i}$ is the mass attenuation coefficient of the element.

The half-value thickness (HVL) of the absorbent material is expressed as the thickness of the absorber required to halve the initial radiation intensity. One tenth $(1 / 10)$ value thickness (TVL) is the thickness required to reduce the high-energy photon intensity that interacts with the material to $1 / 10$ [14-15]. HVL and TVL values were calculated by helping Equation (2) and (3).

$$
\begin{gathered}
H V L=\frac{\operatorname{In} 2}{\mu_{\text {alloy }}}=\frac{0.6931}{\mu_{\text {alloy }}} \\
T V L=\frac{\operatorname{In} 10}{\mu_{\text {alloy }}}=\frac{2.3025}{\mu_{\text {alloy }}}
\end{gathered}
$$

Mfp parameter, 1 (mean free path) average free path was calculated using Equation (4).

$$
\lambda=\frac{1}{\mu_{\text {alloy }}}
$$

\section{RESULTS}

In this manuscript, the absorption parameters of the some shape memory alloys were calculated at the $\mu / \rho, H V L$, TVL and $\lambda$ 5.9-6.1-8-11.2-25-56.543-75-112-149 keV photon energies and listed in Tables 3.1. The $\mu / \rho$ values and absorption parameters of some shape memory alloys were theoretically calculated using WinXCOM data program.

Table-3.1 The Mass Attenuation Coefficient (M/P) Values of Some Shape Memory Alloys At Different Energy

\begin{tabular}{|c|c|c|c|c|c|c|c|c|c|}
\hline \multirow{2}{*}{$\begin{array}{c}\text { Shape Memory } \\
\text { Alloys }\end{array}$} & \multicolumn{9}{|c|}{$\mu / \rho\left(\mathrm{cm}^{2} / \mathrm{gr}\right)$} \\
\cline { 2 - 11 } & $\begin{array}{r}\mathbf{5 . 9} \\
\mathbf{k e V}\end{array}$ & $\begin{array}{c}\mathbf{6 . 1} \\
\mathbf{k e V}\end{array}$ & $\begin{array}{c}\mathbf{8} \\
\mathbf{k e V}\end{array}$ & $\begin{array}{c}\mathbf{1 1 . 2} \\
\mathbf{k e V}\end{array}$ & $\begin{array}{c}\mathbf{2 5} \\
\mathbf{k e V}\end{array}$ & $\begin{array}{c}\mathbf{5 9 . 5 4 3} \\
\mathbf{k e V}\end{array}$ & $\begin{array}{c}\mathbf{7 5} \\
\mathbf{k e V}\end{array}$ & $\begin{array}{c}\mathbf{1 1 2} \\
\mathbf{k e V}\end{array}$ & $\begin{array}{c}\mathbf{1 4 9} \\
\mathbf{k e V}\end{array}$ \\
\hline $55 \%$ Ag-45\%Cd & 490.1 & 449.3 & 220.4 & 89.48 & 10.26 & 5.954 & 3.206 & 1.116 & 0.558 \\
\hline $50 \%$ Au-50\%Cd & 471.9 & 433.4 & 216.3 & 90.14 & 27.31 & 5.360 & 2.919 & 2.504 & 1.229 \\
\hline $82 \% \mathrm{Cu}-14 \% \mathrm{Al}-4 \% \mathrm{Ni}$ & 120.7 & 110.1 & 52.12 & 142.2 & 15.87 & 1.434 & 0.797 & 0.334 & 0.211 \\
\hline
\end{tabular}




\begin{tabular}{|c|l|l|l|l|l|l|l|l|l|}
\hline I International Journal of Technical Research \& Science \\
\hline $85 \% \mathrm{Cu}-15 \% \mathrm{Sn}$ & 185.8 & 169.9 & 82.18 & 153.5 & 17.23 & 2.387 & 1.301 & 0.496 & 0.283 \\
\hline $61 \% \mathrm{Cu}-39 \% \mathrm{Zn}$ & 126.5 & 115.5 & 54.96 & 167.6 & 18.93 & 1.692 & 0.928 & 0.374 & 0.228 \\
\hline $80 \% \mathrm{In}-20 \% \mathrm{Ti}$ & 514.9 & 470.8 & 231.7 & 94.04 & 10.62 & 5.306 & 2.857 & 1.010 & 0.510 \\
\hline $63 \% \mathrm{Ni}-37 \% \mathrm{Al}$ & 116.6 & 106.3 & 49.82 & 105.1 & 11.57 & 1.075 & 0.617 & 0.282 & 0.191 \\
\hline
\end{tabular}

The change of the mass absorption coefficient given in Table 1 according to energy is shown in Fig. 3.1. It is seen that $\mu / \rho$ values are dependent on photon energy and decrease with increasing photon energy. The variation of calculated half value layer, tenth value layer and mean free path for these alloys with respect to the studied photon energy is shown in Figure 3.1-3.4. As can be seen from the graphs drawn, it has been observed that the HVL, TVL and $\lambda$ parameters increase with increasing photon energy.

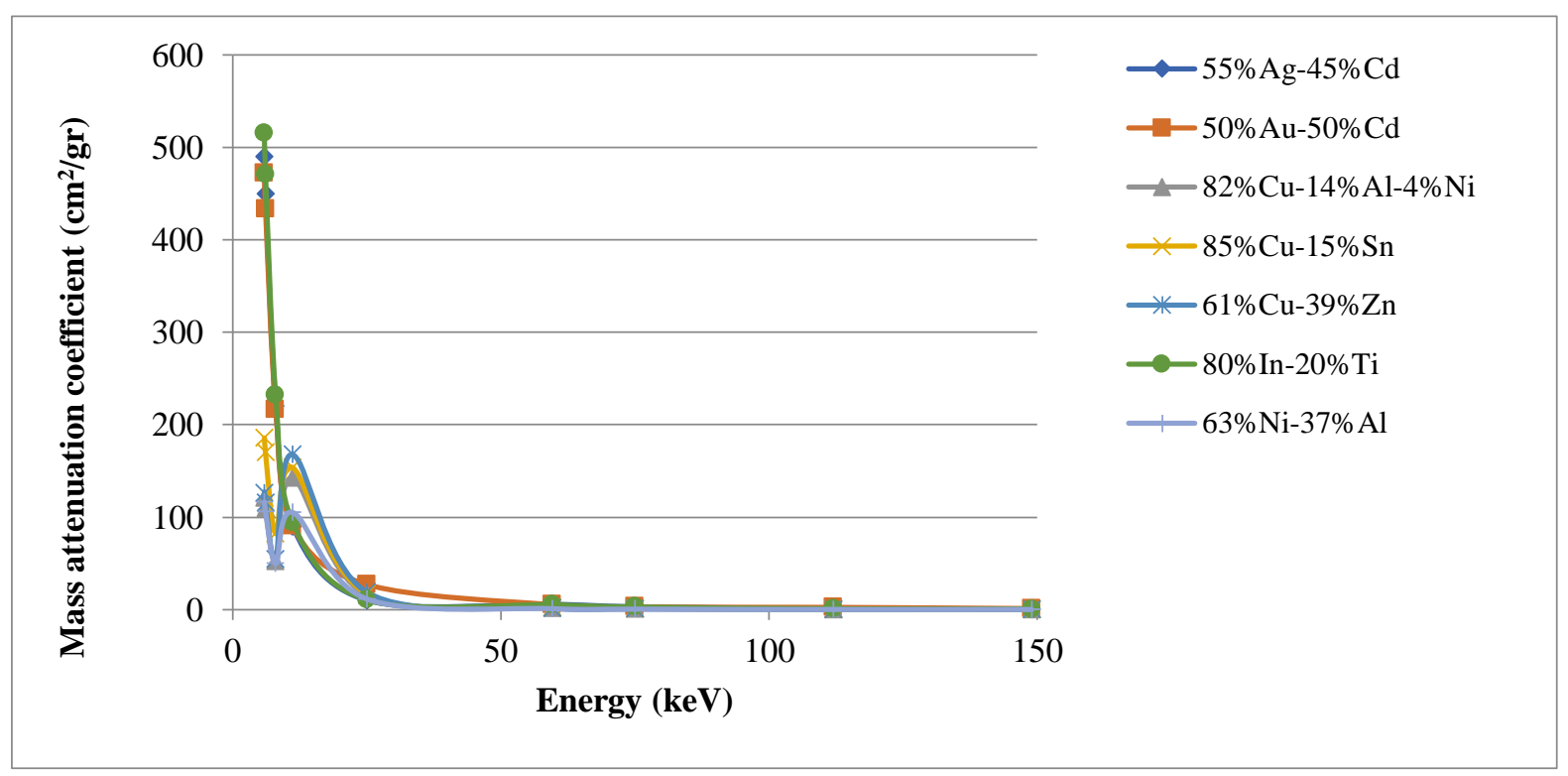

Fig. 3.1 The Change in the Mass Attenuation Coefficient of SMAs different at Energy

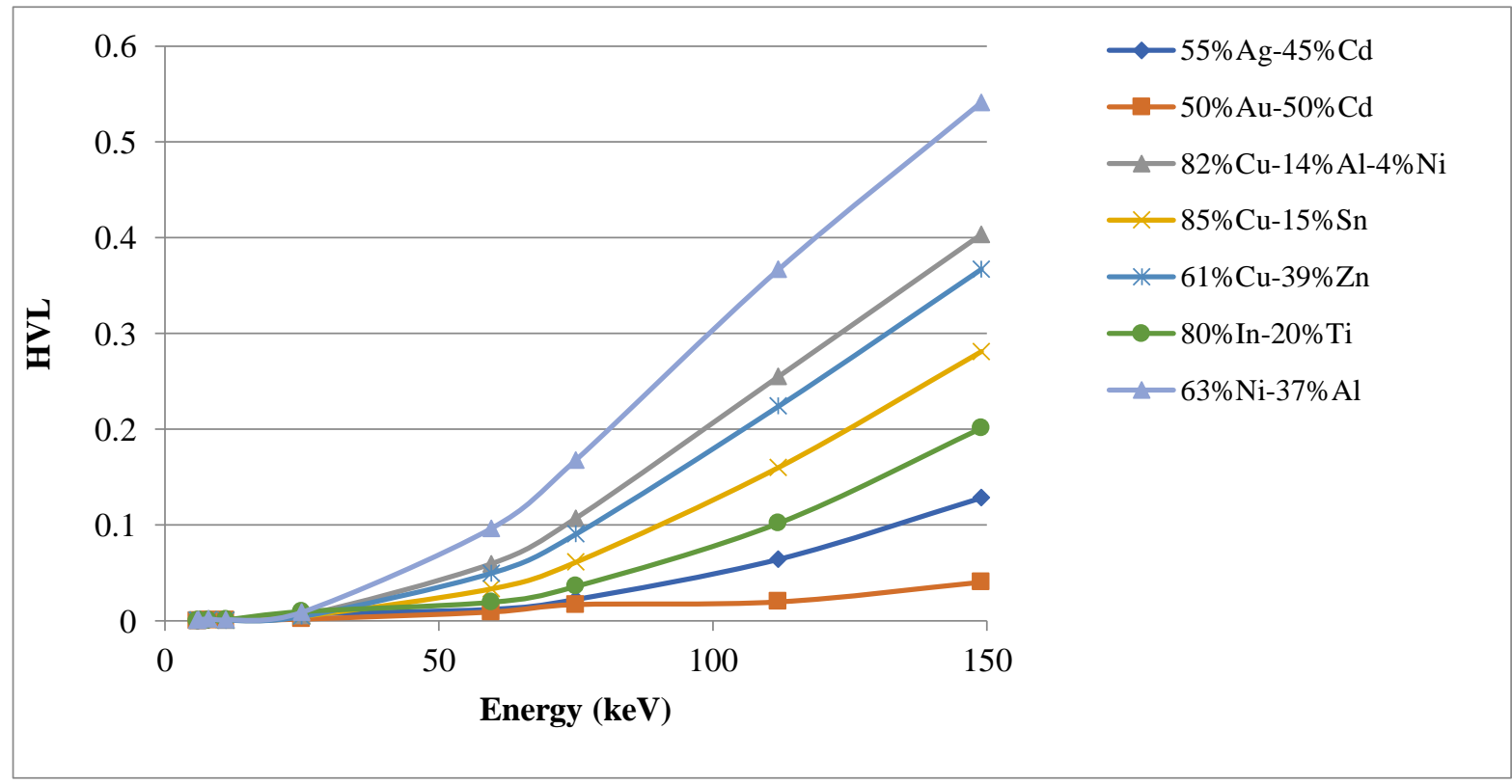

Fig 3.2 Hemi-value Layer (HVL) Change of SMAs different at Energy

DOI Number: https://doi.org/10.30780/IJTRS.V06.I03.002

pg. 16

www.ijtrs.com, www.ijtrs.org

Paper Id: IJTRS-V6-I3-004

Volume VI Issue III, March 2021 @2017, IJTRS All Right Reserved 


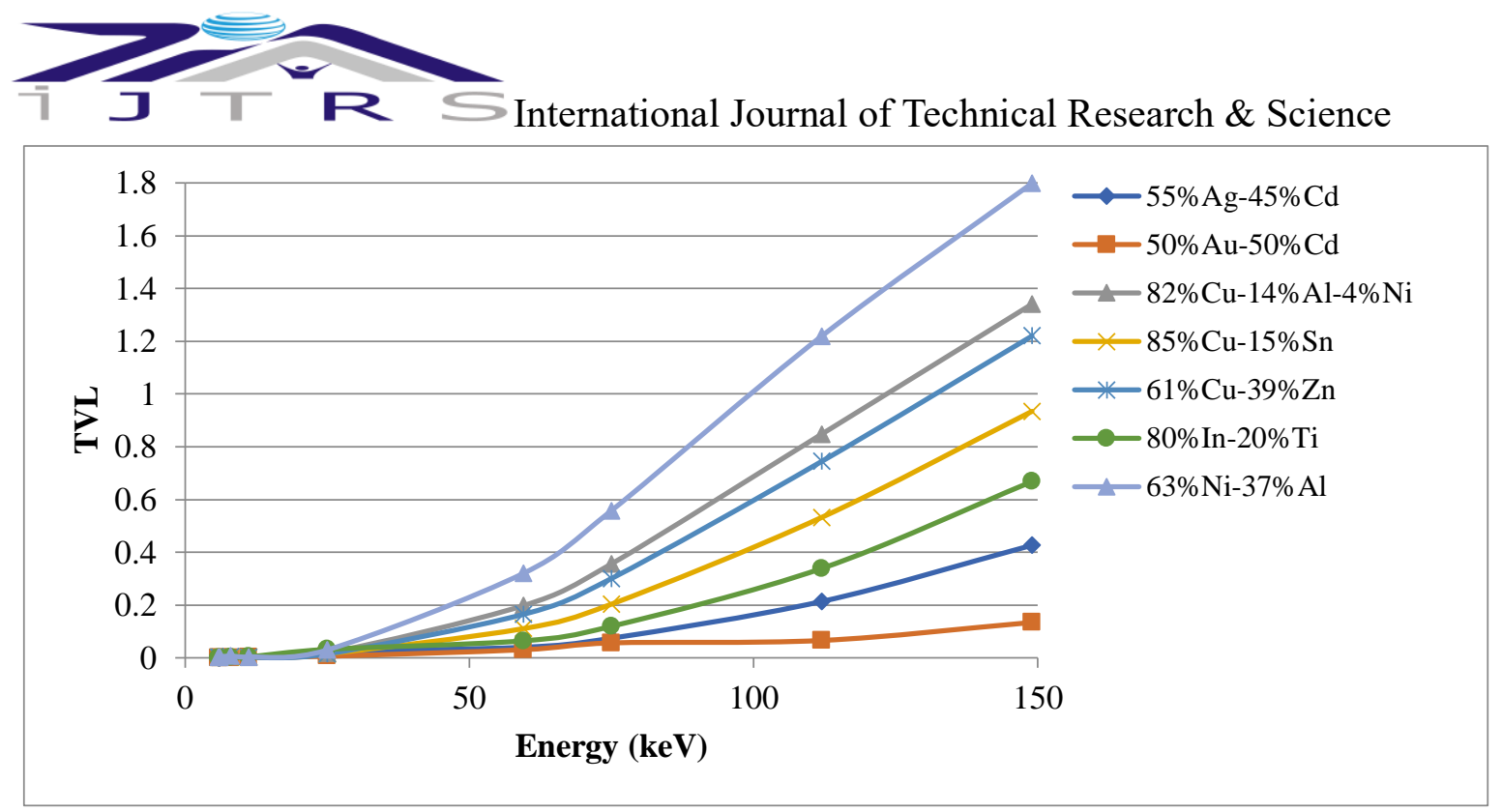

Fig. 3.3 The Change in one tenth (TVL) Value Layer of SMAs different at Energy

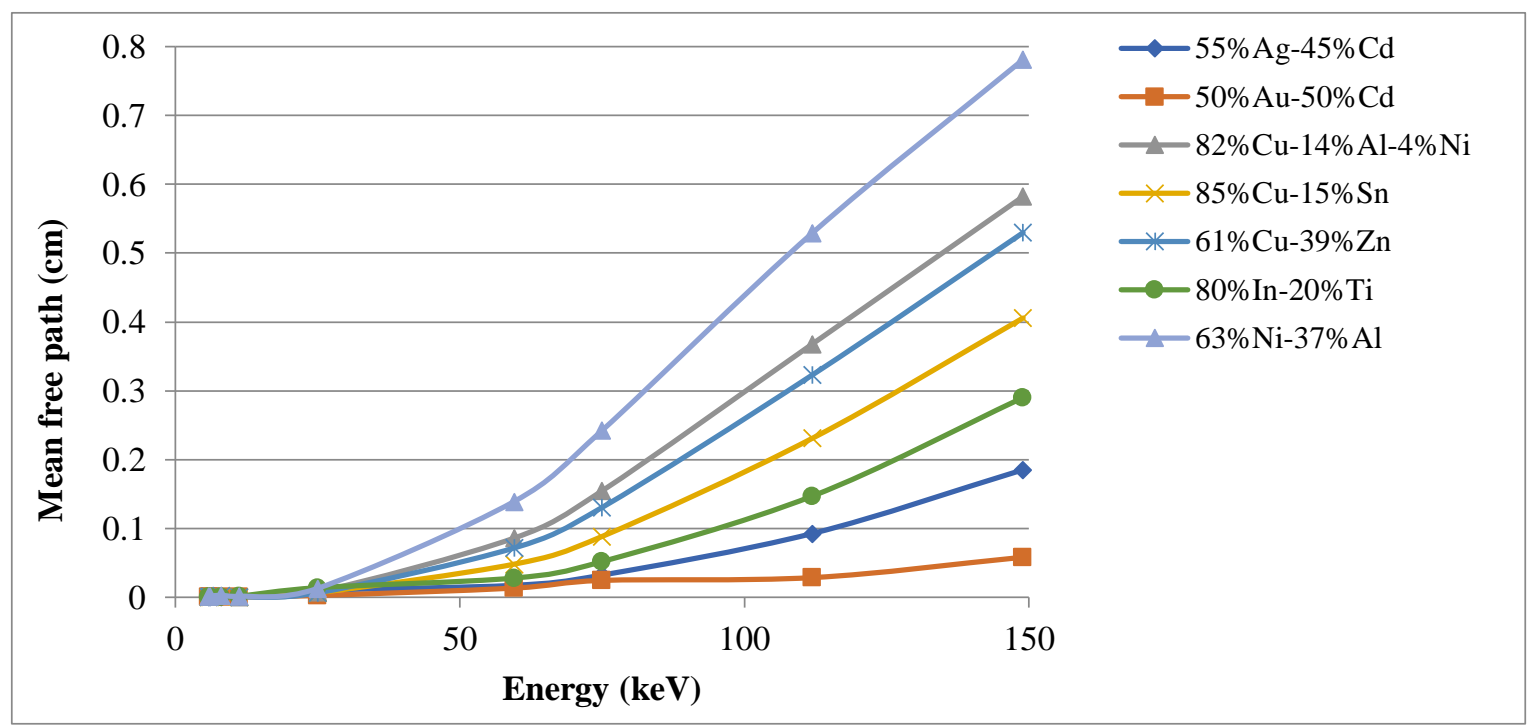

Fig. 3.4 The Change in mean Free Path (Mfp) of SMAs different at Energy

\section{CONCLUSION}

The mass attenuation coefficients, half value layer, tenth value layer, mean free path values some of SMA 5.9-6.1-8-11.2-25-59.543-75-112-149 keV photon energies were calculated with the help of WinXCOM data program. In this research, to obtain sufficient information about $\mu / \rho, \mathrm{HVL}, \mathrm{TVL}$ and $\lambda$ of AgCd, AuCd, CuAlNi, $\mathrm{CuSn}, \mathrm{CuZn}, \mathrm{InTi}, \mathrm{NiAl}$. This alloys have been studied and although it is known that existing alloys have many uses in many areas, they are also used in shielding as an alternative area of use due to their high mass absorption coefficient values.

In this study, the mass absorption coefficients, half-value thickness, tenth-one thickness and mean free path values of some shape memory alloys were theoretically calculated 5.9-6.1-8-11.2-25-59.543-75-112-149 keV energies. The results obtained are given in Table 1 .

It is observed from Table 1 that the mass absorption coefficients of some SMA alloys decrease with increasing energy. It has been observed that the absorption coefficients in the low energy region decrease with increasing energy. The biggest factor in this is that the photoelectric effect is dominant at low energies. It was observed that the mass attenuation coefficients in the medium energy region did not change in large amounts depending on the concentration of the alloy compared to the low energy region, and as a result of the study, it was found that the mass attenuation coefficients increased with the increase atomic number of element. It was observed that the possibility of photon interaction with atom and electron with increasing photon energy decreased. It has been 


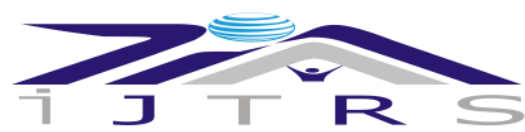

International Journal of Technical Research \& Science

observed that alloys differ according to the photon energy, and it shows itself with the increase in mass absorption coefficients in alloys with $\mathrm{Ag}$ and $\mathrm{Au}$ doped of $\mathrm{Cd}$, where alloys materials containing large atomic numbers absorb the incoming photon strongly. It also expresses the dependence of the $\mu / \rho$ value of this increase on the atomic number when compared to other SMA. For this reason, the current research has been carried out to create a database in order to predict the reactions of the alloys to high-energy photons while the most used SMAs are used in radiated environments in order to be used as a reference in future studies.

This study is important in terms of giving an idea about the radiation interactions of shape memory alloys containing Cd element for use in nuclear technology, radiation protection, building, industry and health fields, and also providing data for the literature.

\section{REFERENCES}

[1] Kumar P.K., Lagoudas D.C. Introduction to shape memory Alloys Springer Science, Business media LLC, 2008. DoI: - 10.1007 / 978 -0-387-47685-8-1.

[2] Jackson, D.F., Hawkes, D.J., X-ray attenuation coefficients of elements and mixtures Phys. Rep.; Vol. 70, pp 169- 233, 1981.

[3] Manohara S.R., Hanagodimath S.M., Studies on effective atomic numbers and electron densities of essential amino acids in the energy range $1 \mathrm{keV}-100 \mathrm{GeV}$, Nuclear Instruments and Methods in Physics Research B, Vol. 258, pp 321-328, 2007.

[4] Kaewkhao J., Laopaiboon J., Chewpraditkul W., Determination of effective atomic numbers and effective electron densities for Cu/Zn alloy, Journal of Quantitative Spectroscopy \& Radioactive Transfer, Vol. 109, pp 1260-1265, 2008.

[5] Nurveren, K., Akdoğan, A., “NiTi Şekil Hafızalı Alaşım Üretimi”, MakinaTek, pp 98-104, 2005.

[6] Akdoğan, A., Nurveren, K., "Şekil Hafızalı Alaşımlar”, Mühendis ve Makina, Vol. 521, pp 35-44, 2003.

[7] Sivakumar, S., Kumar, K., Rao, L., Rao, M., "A Review of the Constitutive Models for Shape Memory Alloys", International Conference on Smart Materials Structures and Systems, Bangalore, India. 2005.

[8] Manohara S.R., Hanagodimath S.M., Gerward L., Studies on effective atomic number, electron density and kerma for some fatty acids and carbohydrates, Physics in Medicine and Biology, Vol. 53, no. 20, pp. 377-386, 2008.

[9] Pawar P.P., Bichile G.K., Studies on mass attenuation coefficient, effective atomic number and electron density of some amino acids in the energy range 0.122-1.330 MeV, Radiation Physics and Chemistry, 92, 22-27, 2013.

[10] Ladhaf B.M., Pawar P.P., Studies on mass energy absorption coefficient and effective atomic energy absorption cross-section for carbohydrates, Radiation Physics and Chemistry, 109, 89-94, 2015.

[11] Gaikwad D.K., Pawar P.P., Selvam T.P., Attenuation cross sections measurements of some fatty acids in the energy range 122-1330 keV. Pramana- J. Phys. 87 (12), 1-7. DOI: 10.1007/s12043-016-1213-y, 2016.

[12] Awasarmol V.V., Gaikwad D.K., Raut S.D. Pawar P.P., Photon interaction study of organic nonlinear optical materials in the energy range 122-1330 keV. Radiation Physics and Chemistry, Vol.130, pp 343-350, 2017.

[13] Hubbell, J.H., Review of photon interaction cross section data in the medical and biological context. Physics in Medicine \& Biology, 44, R1-R22, 1999.

[14] Agar, O., Sayyed, M.I., Akman, F., Tekin, H.O., Kaçal, M.R. An extensive investigation on gamma ray shielding features of Pd/Ag-based alloys. Nuclear Engineering and Technology, Vol. 51, No.3, pp 853-859, 2019.

[15] Akkaş A., Determination of the Tenth and Half Value Layer Thickness of Concretes with Different Densities, Acta Physica Polonica A, Vol. 129, pp 770-772, 2016. 